\title{
Facile Synthesis of Gd-Functionalized Gold Nanoclusters as Potential MRI/CT Contrast Agents
}

\author{
Wenjun Le ${ }^{1,+}$, Shaobin Cui ${ }^{1,+}$, Xin Chen $^{1}$, Huanhuan Zhu ${ }^{1}$, Bingdi Chen ${ }^{1,2, *}$ \\ and Zheng Cui ${ }^{1,3, *}$ \\ 1 The Institute for Translational Nanomedicine, Shanghai East Hospital, The Institute for Biomedical \\ Engineering \& Nano Science, Tongji University School of Medicine, Shanghai 200120, China; \\ lwj2120@126.com (W.L.); cbin1991@hotmail.com (S.C.); xinchen513@yahoo.com (X.C.); \\ zhuhuanhuan132@163.com (H.Z.) \\ 2 State Key Lab of Silicon Materials, Zhejiang University, Hangzhou 310027, China \\ 3 Department of Pathology, Wake Forest University School of Medicine, Winston-Salem, NC 28780, USA \\ * Correspondence: inanochen@tongji.edu.cn (B.C.); zheng_cui@tongji.edu.cn (Z.C.); \\ Tel.: +86-21-6598-3706 (B.C.); +86-21-6598-8029 (Z.C.) \\ + These authors contributed equally to this work.
}

Academic Editor: Yurii Gun'ko

Received: 4 February 2016; Accepted: 28 March 2016; Published: 9 April 2016

\begin{abstract}
Multi-modal imaging plays a key role in the earlier detection of disease. In this work, a facile bioinspired method was developed to synthesize Gd-functionalized gold nanoclusters (Gd-Au NCs). The Gd-Au NCs exhibit a uniform size, with an average size of $5.6 \mathrm{~nm}$ in dynamic light scattering (DLS), which is a bit bigger than gold clusters (3.74 nm, DLS), while the fluorescent properties of Gd-Au NCs are almost the same as that of Au NCs. Moreover, the Gd-Au NCs exhibit a high longitudinal relaxivity value (r1) of $22.111 \mathrm{~s}^{-1}$ per $\mathrm{mM}$ of $\mathrm{Gd}$ in phosphate-buffered saline (PBS), which is six times higher than that of commercial Magnevist (A complex of gadolinium with a chelating agent, diethylenetriamine penta-acetic acid, Gd-DTPA, $r 1=3.56 \mathrm{mM}^{-1} \cdot \mathrm{s}^{-1}$ ). Besides, as evaluated by nano single photon emission computed tomography (SPECT) and computed tomography (CT) the Gd-Au NCs have a potential application as CT contrast agents because of the Au element. Finally, the Gd-Au NCs show little cytotoxicity, even when the Au concentration is up to $250 \mu \mathrm{M}$. Thus, the Gd-Au NCs can act as multi-modal imaging contrast agents.
\end{abstract}

Keywords: gold nanocluster; bovine serum albumin; contrast agent; magnetic resonance imaging; computed tomography

\section{Introduction}

Multi-modality imaging is now commonplace in clinical practice [1], especially in the field of nuclear medicine, positron emission tomography / computed tomography (PET/CT) and single-photon emission computed tomography (SPECT)/CT [2]. The current method relies on positron emission tomography (PET), which is expensive and exposes people to radiation, and these are undesirable features for a population screening method [3]. However, the conundrum of modality selection in clinical diagnostic imaging is that modalities with the highest sensitivity have relatively poor resolution, while those with high resolution have relatively poor sensitivity [4].

Magnetic resonance imaging (MRI), as a common and cheaper imaging technology since 1970s, has become a powerful imaging modality for clinical diagnostic imaging, depending on the advantages of being non-invasive, having no ionizing radiation, and having unlimited depth of tissue penetration and high spatial resolution, especially for soft tissues [5-8]. The most extensively currently used contrast agents in the clinic are paramagnetic gadolinium $(\mathrm{Gd})$ chelates, such as Gd-DTPA (Magnevist ${ }^{\circledR}$, Schering AG, Berlin, Germany). Gd chelates can change the signal intensity by shortening the 
longitudinal relaxation time (T1) of water molecules [9]. Unfortunately, due to fast renal clearance resulting from the low molecular weight, the biomedical applications of Gd chelates are discouraged by the short intrinsic response time and non-specificity to target organs [10]. Therefore, designing and developing an alternative approach with non-toxicity, prolonged residence time, and specific distribution has become critically important [11-13].

Computed tomography (CT) has the advantages of rapid image acquisition and high contrast and spatial resolution; the advent of it has revolutionized diagnostic radiology [14,15]. However, it also has some limitations. For instance, it is an increasing source of radiation exposure to patients. In addition, it has a superb three-dimensional (3D) resolution for structure imaging but is short on tissue sensitivity for functional imaging [16]. Multi-modality imaging with two or more imaging modalities can therefore allow the integration of strengths of individual modalities while overcoming their limitations $[17,18]$. CT and MRI, for example, provide unparalleled structural detail in anatomical imaging technologies [19].

Recently, the development of new multi-functional bionanocomposites shows a promising research topic due to the properties inherent from the biopolymers with biocompatibility and biodegradability [20]. Bovine serum albumin (BSA), as a common and economical available biopolymer, has been widely applied in the preparation of bionanocomposites (e.g., Au nanoclusters [21], CdSe [22], $\mathrm{Cu}$ nanoclusters [23,24], etc.) for in vivo bioapplications during the past few years. The preparations of the above nanoclusters have a lot of advantages, such as milder reaction conditions, facile processing, good reproducibility, biocompatibility, and robust stability. Inspired by the above-mentioned reports, in this study, we report a facile, one-pot route for the preparation of Gd-Au nanoclusters (Gd-Au NCs) through an albumin-mediated strategy. The obtained Gd-Au NCs exhibited a pronounced elevation of higher longitudinal relaxivity $\left(\mathrm{r} 1=22.111 \mathrm{mM}^{-1} \cdot \mathrm{s}^{-1}\right)$ than Magnevist $\left(\mathrm{r} 1=3.56 \mathrm{mM}^{-1} \cdot \mathrm{s}^{-1}\right)$. Consequently, it can potentially be employed for multi-modal imaging contrast agents.

\section{Results and Discussion}

\subsection{Preparation of $G d-A u N C s$}

In bioinspired biomineralization, the biomacromolecules are used to collect and transport raw materials and assemble them into ordered composites with consistency and uniformity in an aqueous environment under mild conditions [13]. BSA as a biomacromolecule is commonly employed as a template in biomineralization. The molecular structure of BSA includes lots of disulfide bonds (among the 17 available per molecular), and these bonds have a strong affinity with the surface of metal atoms [25]. At the same time, some amino acids (e.g., tyrosine) of BSA possess strong reducibility under alkaline conditions. In the experiment, $\mathrm{Au}$ NCs were synthesized according to an improved "green" synthetic route [21]. The $\mathrm{pH}$ values of the solutions were adjusted to 12 to trigger the reduction capability of the responsible amino acids, and then the solutions were maintained at $37^{\circ} \mathrm{C}$ for $12 \mathrm{~h}$ to ensure the complete reduction of $\mathrm{HAuCl}_{4}$.

In the synthesis of Gd-Au NCs, aqueous gadolinium chloride solution is introduced to the above reaction system. Briefly, aqueous gadolinium chloride solution was mixed with $\mathrm{HAuCl}_{4}$ solution under vigorous stirring. Then, BSA solution was added to the mixture under the same condition. Ten minutes later, $\mathrm{NaOH}$ solution was utilized to adjust the $\mathrm{pH}$ values of the reaction solution, and the mixture was subsequently stirred at $37^{\circ} \mathrm{C}$ (water bath). The color of the solution would change from light yellow to deep brown, which indicated that the Gd-Au nanoclusters were formed and they tended to be stable. Purification of Gd-Au NCs was firstly performed by dialysis to remove the small molecules, included some metal ions. The residue was subsequently freeze-dried from liquid to solid. Finally, the powder was dispersed in phosphate-buffered saline (PBS, $0.01 \mathrm{M}, \mathrm{pH}$ 7.4) and stored at $4{ }^{\circ} \mathrm{C}$ for further study.

\subsection{Characterization of $G d-A u$ NCs}

The Gd-Au NCs were synthesized in one step, and characterized by transmission electron microscopy (TEM), dynamic light scattering (DLS), fluorescent emission spectra, and ultraviolet-visible 
(UV-vis) spectroscopy, etc. As shown in Figure 1, the TEM image (Figure 1a) and the results of DLS (Figure 1b) consistently disclose that Gd-Au NCs exhibit a uniform size, with an average hydrodynamic diameter of $5.6 \mathrm{~nm}$, which is a bit bigger than that of gold clusters ( $3.74 \mathrm{~nm}$, DLS) reported in the literature [26]. The size of the nanoclusters increased, and this is likely due to the result of the Gd ion being directly involved in the formation of clusters. Therefore, in this study, we subsequently investigated the influence of gadolinium salt on the fluorescent properties of Au NCs (Figure 2). UV-vis absorption and the corresponding fluorescent emission spectra of Au NCs and Gd-Au NCs are shown in Figure 2a,b. The as-prepared nanoclusters have a photoemission peak at $\sim 670 \mathrm{~nm}$. The bright photograph and the corresponding fluorescent photograph of Au NCs and Gd-Au NCs are shown in Figure 2c,d. No obvious change in fluorescence properties was observed between Au NCs and Gd-Au $\mathrm{NCs}$ in solutions under the same concentrations of $\mathrm{Au}$ from the inductively coupled plasma mass spectrometry (ICP-MS) measurement. In addition, we found that the molar ratio of Au:Gd elements in Gd-Au NCs solutions was 1:1.09.
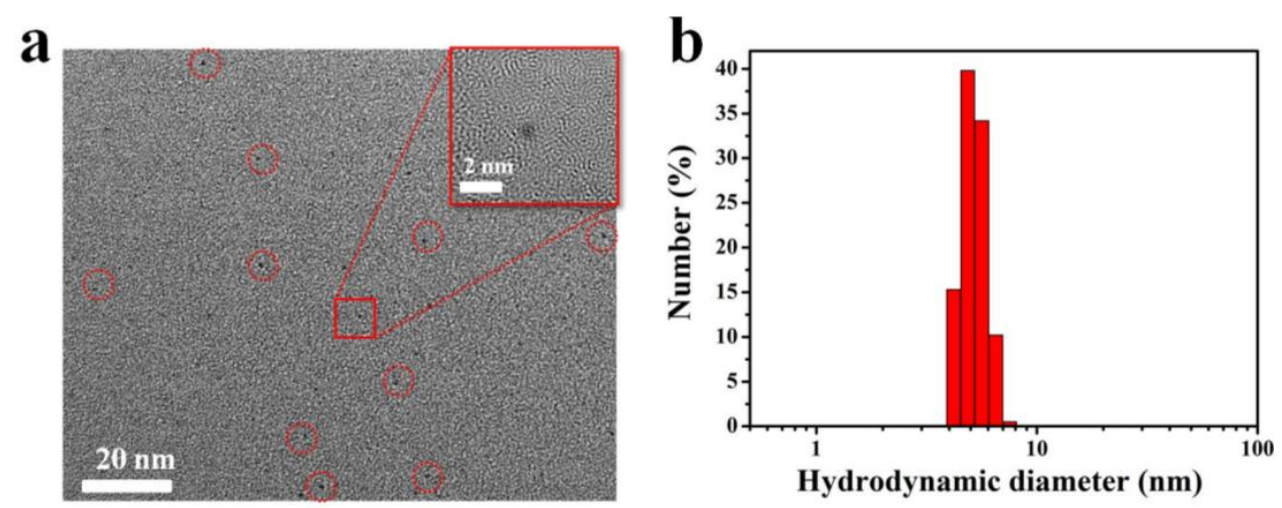

Figure 1. (a) Transmission electron microscopy image of as-prepared Gd-Au nanoclusters; (b) The result of dynamic light scattering.

a

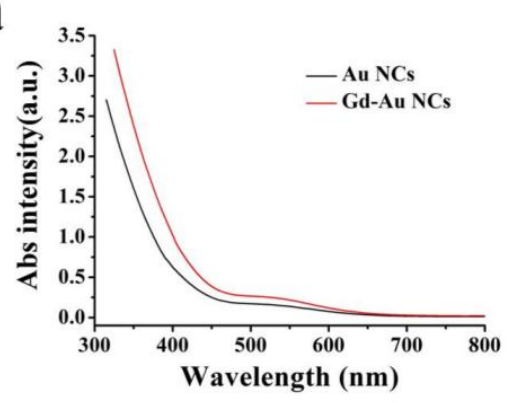

C

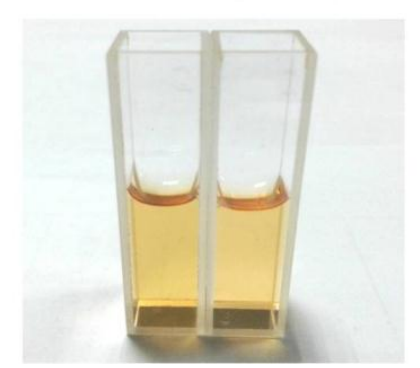

b

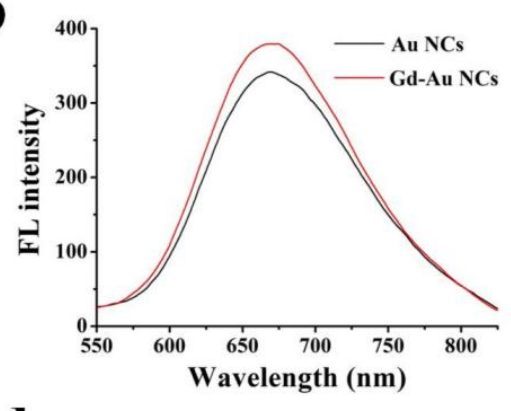

d

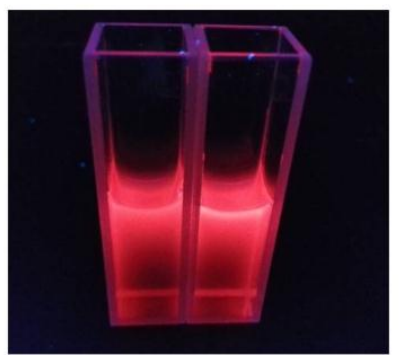

Figure 2. (a) Ultraviolet-visible absorption spectra of Au nanoclusters (NCs) (black curve) and Gd-Au NCs (red curve), Abs (Absorbance), a.u. (Absorbance Unit); (b) The corresponding fluorescent emission spectra of Au NCs (black curve) and Gd-Au NCs (red curve); (c) Bright photograph of Au NCs (left) and Gd-Au NCs (right); (d) The corresponding fluorescent photograph of Au NCs (left) and Gd-Au NCs (right) was taken under a porTable 365 nm UV-lamp (Min hang, Shanghai, China). 
The oxidation states of the Gd-Au NCs and Au NCs were determined by X-ray photoelectron spectroscopy (XPS). The XPS measurement was performed on a Perkin-Elmer PHI5300 spectrometer (Waltham, MA, USA). Figure S1 demonstrates the photoelectron spectra of the Gd-Au NCs and Au NCs. The $4 f_{7 / 2}$ and $4 f_{5 / 2}$ binding energy values of gold appeared at $83.2 \mathrm{eV}$ and $87.1 \mathrm{eV}$ (Figure S1a), respectively. In addition to the gadolinium peak of the $\mathrm{Gd} 4 \mathrm{~d}$ region (Figure S1b), the region also matched well with the data reported on $\mathrm{Gd}$-doped $\mathrm{CeO}_{2}$ [27]. If they were only chelated to BSA on the Au surface, it would involved a spin-orbit coupled $3 \mathrm{~d} 5 / 2$ and $3 \mathrm{~d}_{3 / 2}$ doublet with a binding energy position of $1186 \mathrm{eV}$ and $1218 \mathrm{eV}$ [28], respectively. Meanwhile, the XPS of Au NCs are shown in Figure $\mathrm{S} 1 \mathrm{c}, \mathrm{d}$. The $4 \mathrm{f}_{7 / 2}$ and $4 \mathrm{f}_{5 / 2}$ binding energy values of gold appeared at $84.2 \mathrm{eV}$ and $87.6 \mathrm{eV}$ (Figure S1a), respectively. In addition, there was no characteristic peak for the $4 \mathrm{~d}$ region of Gd in XPS of Au NCs. The above results may indicate that the gadolinium was successfully involved in the formation of Au clusters.

\subsection{MRI/CT in Vitro}

To evaluate the capacity of Gd-Au NCs as an effective the longitudinal relaxation time (T1)-weighted MRI contrast agent, the longitudinal (T1) and transverse (T2) relaxation times were measured with a 1.5 T NMR analyzer (Milton, ON, Canada) at different Gd concentrations (9.325, $18.75,37.5,75,150 \mu \mathrm{M})$ from the ICP-MS measurement, respectively. As shown in Figure 3a, the Gd-Au NCs exhibited a high $\mathrm{r} 1$ value of $22.111 \mathrm{~s}^{-1}$ per $\mathrm{mM}$ of Gd in PBS, which is six times higher than that of commercial Magnevist (Gd-DTPA, $\mathrm{r} 1=3.56 \mathrm{mM}^{-1} \cdot \mathrm{s}^{-1}$ ) [12]. The significant increasing may be due to the favorable water solubility, the small size, and the confined tumbling in the biomacromolecule, resulting in a longer rotational correlation time $[29,30]$. In addition to the improved longitudinal relaxivity $(\mathrm{r} 1)$, the relatively low ratio $(\mathrm{r} 2 / \mathrm{r} 1=1.73<3)$ is beneficial in producing a desired $\mathrm{T} 1$ positive contrast effect [31]. To explore the potential of Gd-AuNCs as MRI contrast agents, different Gd concentrations $(0,0.08,0.16,0.24,0.32,0.40 \mathrm{mM})$ were evaluated by a $3.0 \mathrm{~T}$ clinical MR scanner (GE, Milwaukee, WI, USA) at $25^{\circ} \mathrm{C}$. T1-weighted magnetic resonance (MR) images and the corresponding signal intensity in Figure $3 \mathrm{~b}$ further confirmed that the Gd-Au NCs exhibited an enhanced T1 signal, demonstrating that they can act as a highly efficient T1-enhanced MR contrast agent in vitro.
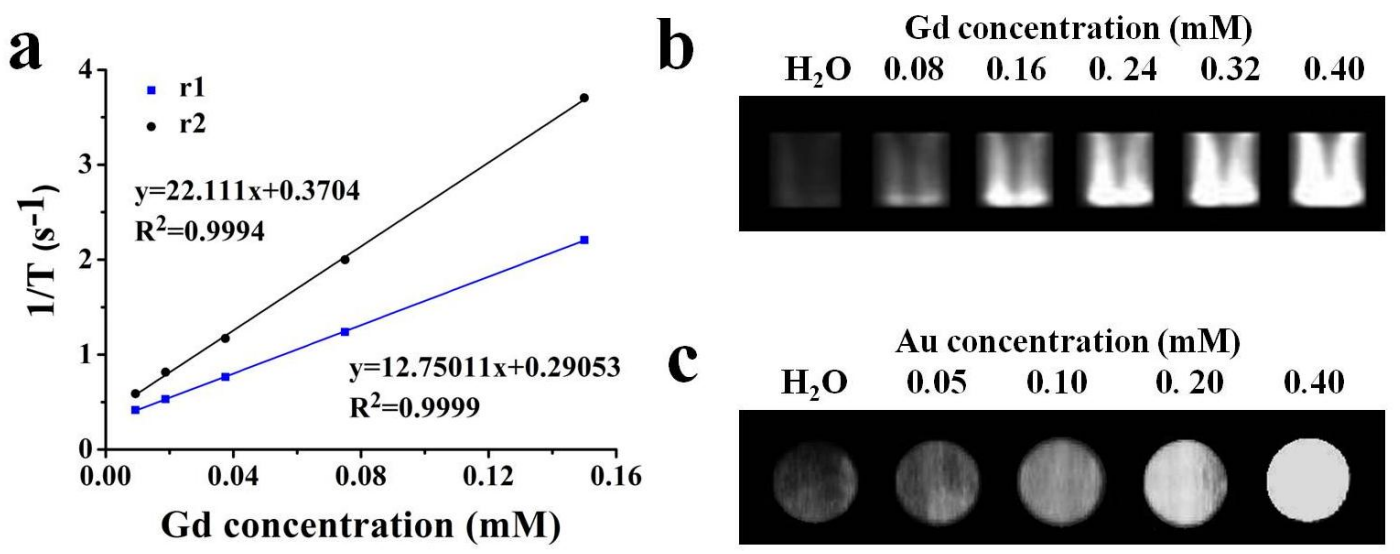

Figure 3. (a) Longitudinal (T1) and transverse (T2) relaxation times of Gd-Au NCs (the slopes response to the longitudinal relaxivity value r1( blue) and transverse relaxivity value $\mathrm{r}$ (black)); (b) Magnetic resonance images of the Gd-Au NCs with Gd concentrations ranging from 0.08 to $0.40 \mathrm{mM}$ and $\mathrm{H}_{2} \mathrm{O}$; (c) Computed tomography of the Gd-Au NCs containing various Au concentrations and $\mathrm{H}_{2} \mathrm{O}$.

The Au element has higher X-ray attenuation than iodine due to its higher atomic number and electron density [19]. Furthermore, to confirm the feasibility of Gd-Au NCs for CT contrast agents, different Au concentrations $(0,0.05,0.1,0.20,0.40 \mathrm{mM})$ from the ICP-MS measurements were evaluated by Nano SPECT/CT (Washington, DC, USA). As shown in Figure 3c, the as-prepared Gd-Au NCs 
have a higher signal than $\mathrm{H}_{2} \mathrm{O}$, and as the concentration of gold increased, its corresponding CT signal gradually improved, which reveals that the Gd-Au NCs can also be a potential CT contrast agent in vitro.

\subsection{In Vitro Cytotoxicity}

The cytotoxicity of Gd-Au NCs was evaluated via cell counting kit-8 (CCK-8) assay by incubating the breast cancer cell line (MCF-7) with Au NCs and Gd-Au NCs at various concentrations of Au for $24 \mathrm{~h}$, respectively. The CCK-8 results in Figure 4 indicated that the Gd-Au NCs showed little cytotoxicity against MCF-7 cells, even at Au concentrations up to $250 \mu \mathrm{M}$. Also, there is no significant difference in cell viability between Au NCs and Gd-Au NCs. This at least indicated that the gadolinium ions have a strong bond with BSA molecules and will not obviously increase the cytotoxicity of nanoclusters in the cell culture system. These results showed that Gd-Au NCs were of low toxicity and safe against the MCF-7 cells at the test concentrations. This was in accordance with the non-toxicity, low immunogenicity, and good biocompatibility and biodegradability of both Au NCs and Gd-BSA as previously reported in literature $[13,28]$.

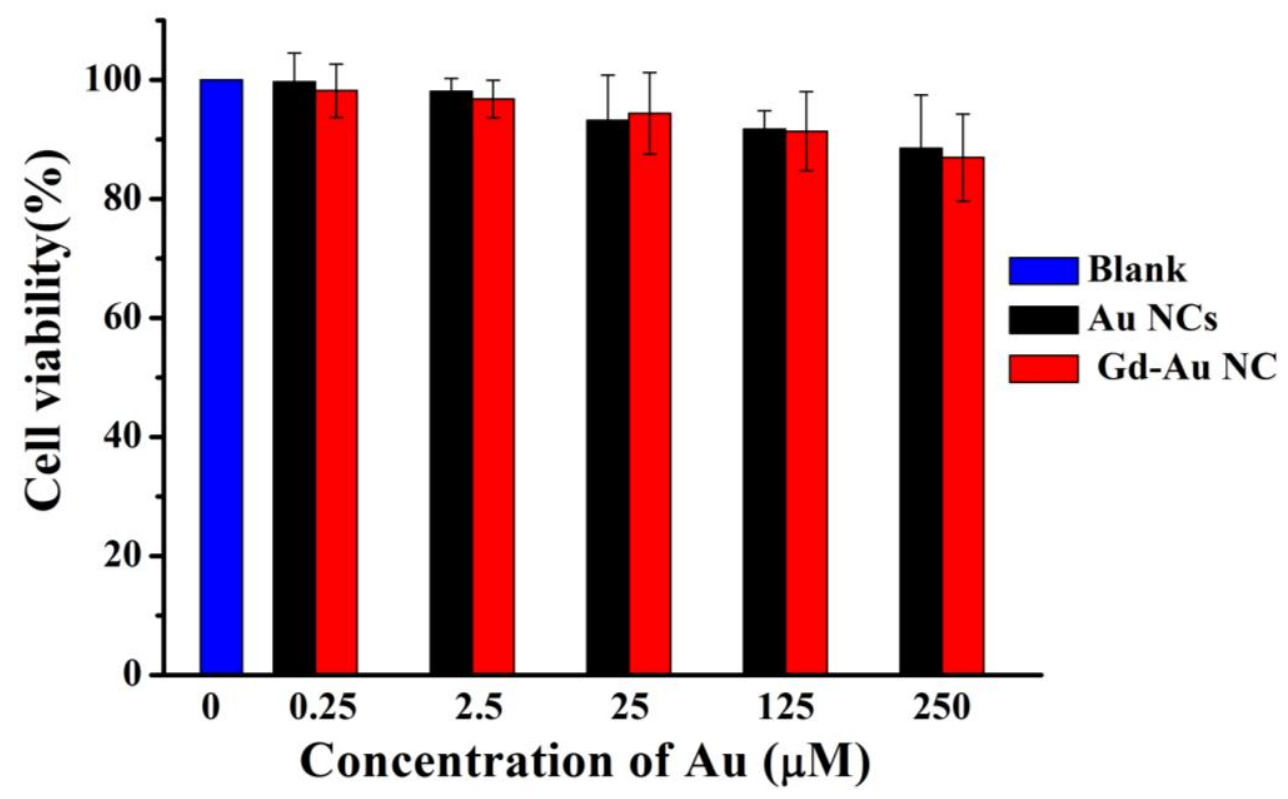

Figure 4. In vitro cytotoxicity of Au NCs (black) and Gd-Au NCs (red) against breast cancer cell line (MCF-7) after $24 \mathrm{~h}$.

\section{Materials and Methods}

\subsection{Materials}

All initial reagents were obtained commercially and used as received. Albumin from bovine serum (BSA) was purchased from Sigma-Aldrich (Louis, MO, USA). The $\mathrm{HAuCl}_{4}$ were purchased from Guoyao Reagent Corporation (Shanghai, China). MCF-7 cell line was purchased from Shanghai Institute of Cell Biology (Shanghai, China). Dulbecco's modified eagle's medium (DMEM), Fetal bovine serum (FBS), Phosphate-buffered saline (PBS) and 0.25\% Trypsin-EDTA were purchased from Gibco Corp (Grand Island, NY, USA). Millipore ultrapure water (Billerica, MA, USA) $(18.2 \mathrm{M} \Omega \cdot \mathrm{cm}$ resistivity at $25^{\circ} \mathrm{C}$ ) was used throughout the entire experiments.

\subsection{Preparation of $G d-A u N C s$}

In a typical experiment, aqueous gadolinium chloride solution $(0.15 \mathrm{~mL}, 500 \mathrm{mM})$ was added to $\mathrm{HAuCl}_{4}$ solution $(5 \mathrm{~mL}, 10 \mathrm{mM})$ slowly under vigorous stirring. Then BSA solution $(5 \mathrm{~mL}, 50 \mathrm{mg} / \mathrm{mL})$ 
was added to the mixture under vigorous. Ten minutes later, $\mathrm{NaOH}$ solution $(0.75 \mathrm{~mL}, 1 \mathrm{M})$ was introduced under ultrasonic dispersion and the mixture was continuously stirred at $37^{\circ} \mathrm{C}$ for $12 \mathrm{~h}$ under nitrogen. During this period, the color of the solution changed from light yellow to light brown, and then to deep brown. Purification of Gd-Au NCs was performed by dialysis to remove the small molecules and was freeze-dried from liquid to solid. Finally, the powder was dispersed in PBS (0.01 M, $\mathrm{pH}$ 7.4) and stored at $4{ }^{\circ} \mathrm{C}$ for further study.

BSA-stabilized Au clusters (Au NCs) were prepared according to an improved 'green' synthetic route [21].

\subsection{Characterization of $G d-A u N C s$}

Transmission electron microscopy (TEM) images of Gd-Au NCs were obtained using a JEM-2100F electron microscope (JEOL Ltd., Tokyo, Japan) working at $200 \mathrm{kV}$. The nanoparticles were dispersed in deionized water (DIW) and dried onto carbon-coated copper grids. Then the air-dried samples were directly observed by electron microscope. The hydrodynamic diameter analyses of the aqueous were performed on a laser light scattering system (JEM Zetasizer Nano-ZS90, Great Malvern, England, UK). UV-vis absorption and fluorescent emission spectra were measured by Cary 50 spectrophotometer (Varian, Palo Alto, CA, USA) and F-182 4500 spectrophotometer (Hitachi, Chiyoda, Tokyo, Japan), respectively. The concentration of Gd/Au was measured with ICP-AES (P-4010, Hitachi, Chiyoda, Tokyo, Japan).

\subsection{Relaxometry and MRI in Vitro}

The longitudinal (T1) and transverse (T2) relaxation times of Gd-Au NCs were measured with a $1.41 \mathrm{~T}$ minispec mq $60 \mathrm{NMR}$ Analyzer (Bruker, Germany) at $37^{\circ} \mathrm{C}$. The MR phantom images in vitro were acquired using a 3.0 T Sigma scanner (GE, Milwaukee, WI, USA). The T1-weighted MR images of Gd-Au NCs were obtained with different Gd concentrations $(0,0.08,0.16,0.24,0.32,0.40 \mathrm{mM}) \mathrm{using}$ Tl-weighted pulse sequences, respectively. The measurement parameters were as follows: T1-weighted sequence, spin echo (SE), the repetition time (TR) and the echo time (TE) =500/18.2 ms), matrix acquisition $=90 \times 90$, number of complex samples $(\mathrm{NS})=2$, field-of-view $(\mathrm{FOV})=80 \mathrm{~mm} \times 80 \mathrm{~mm}$, slices $=1$, slice width $=5.0 \mathrm{~mm}$, slice gap $=0.55 \mathrm{~mm}, 0.55 \mathrm{~T}, 32.0^{\circ} \mathrm{C}$. Relaxivity values of $\mathrm{r} 1$ and $\mathrm{r} 2 \mathrm{were}$ calculated by fitting the $1 / \mathrm{T} 1$ and $1 / \mathrm{T} 2$ relaxation time $\left(\mathrm{s}^{-1}\right)$ versus $\mathrm{Gd}$ concentration $(\mathrm{mM})$ curves.

\subsection{In Vitro Cytotoxicity}

MCF-7 cell line was cultured in a $37^{\circ} \mathrm{C}$ incubator with $5 \% \mathrm{CO}_{2}$ in Dulbecco's Modified Eagle Medium (DMEM) supplemented with 10\% FBS, streptomycin at $100 \mathrm{mg} / \mathrm{mL}$ and penicillin at $100 \mathrm{U} / \mathrm{mL}$. The in vitro cytotoxicity of Gd-Au NCs was measured using a standard cell counting kit-8 (CCK-8) assay. Typically, MCF-7 cells (5 × 103/well) were seeded into a 96-well plate (three parallel holes per group), and incubated in the culture medium for $12 \mathrm{~h}$ at $37^{\circ} \mathrm{C}$ under $5 \% \mathrm{CO}_{2}$. The culture medium was then removed, and cells were incubated with fresh culture medium containing $100 \mu \mathrm{L}$ of $\mathrm{Au}$ NCs and Gd-Au NCs at varied Au concentrations $(0.25 \mu \mathrm{M}, 2.5 \mu \mathrm{M}, 25 \mu \mathrm{M}, 125 \mu \mathrm{m}, 250 \mu \mathrm{M})$ at $37{ }^{\circ} \mathrm{C}$ under $5 \% \mathrm{CO}_{2}$ for additional $24 \mathrm{~h}$, respectively. Then $10 \mu \mathrm{L}$ of CCK-8 agentia $(5 \mathrm{mg} / \mathrm{mL})$ was added into the plates and incubating cells for further $3 \mathrm{~h}$. In the end, the OD450 value (Absolute values) of each well was measured using the multifunction microplate reader (Tecan infinite M200 Pro, Tecan Group Ltd., Männedorf, Switzerland).

\section{Conclusions}

In conclusion, we synthesized Gd-Au NCs using bioinspired biomineralization. The particle size of Gd-Au NCs is a bit bigger than that of Au NCs because of the Gd involvement in the formation of clusters, while the Gd-Au NCs exhibit excellent fluorescent properties that are almost the same as that of $\mathrm{Au}$ NCs. The in vitro MRI results show that the r1 value of the Gd-Au NCs is six times higher than that of commercial Magnevist (Gd-DTPA), which is mainly due to the favorable water solubility, the 
small size, and the confined tumbling in a biomacromolecule. Furthermore, the Gd-Au NCs can be used as a CT contrast agent because of the Au element, as evaluated by Nano SPECT/CT. Moreover, the Gd-Au NCs show non-toxicity and good biocompatibility. All these results indicate that Gd-Au NCs are promising for use as a multi-modal imaging contrast agent.

Supplementary Materials: The following are available online at http://www.mdpi.com/2079-4991/6/4/65/s1.

Acknowledgments: This work was supported by Natural Science Foundation of China (51302190), Specialized Research Fund for the Doctoral Program of Higher Education (20130072120029) and the Fundamental Research Funds for the Central Universities.

Author Contributions: Wenjun Le conducted parts of the experiments and prepared the manuscript. Shaobin Cui, Xin Chen and Huanhuan Zhu conducted parts of the experiments and edited the draft. Bingdi Chen, Zheng Cui advised the work and modified the manuscript.

Conflicts of Interest: The authors declare no conflict of interest.

\section{References}

1. Cherry, S.R. Multimodality imaging: Beyond PET/CT and SPECT/CT. Semi. Nucl. Med. 2009, 39, 348-353. [CrossRef] [PubMed]

2. Culver, J.; Akers, W.; Achilefu, S. Multimodality molecular imaging with combined optical and SPECT/PET modalities. J. Nucl. Med. 2008, 49, 169-172. [CrossRef] [PubMed]

3. Cheng, K.K.; Chan, P.S.; Fan, S.; Kwan, S.M.; Yeung, K.L.; Wáng, Y.X.J.; Chow, A.H.L.; Wu, E.X.; Baum, L. Curcumin-conjugated magnetic nanoparticles for detecting amyloid plaques in Alzheimer's disease mice using magnetic resonance imaging (MRI). Biomaterials 2015, 44, 155-172. [CrossRef] [PubMed]

4. Louie, A. Multimodality imaging probes: Design and challenges. Chem. Rev. 2010, 110, 3146-3195. [CrossRef] [PubMed]

5. Brammer, M.J.; Bullmore, E.T.; Simmons, A.; Williams, S.C.R.; Grasby, P.M.; Howard, R.J.; Woodruff, P.W.R.; Rabe-Hesketh, S. Generic brain activation mapping in functional magnetic resonance imaging: A nonparametric approach. Magn. Reson. Imag. 1997, 15, 763-770. [CrossRef]

6. Weissleder, R.; Pittet, M.J. Imaging in the era of molecular oncology. Nature 2008, 452, 580-589. [CrossRef] [PubMed]

7. Taylor-Pashow, K.M.; Rocca, J.D.; Lin, W. Mesoporous silica nanoparticles with co-condensed gadolinium chelates for multimodal imaging. Nanomaterials 2011, 2, 1-14. [CrossRef] [PubMed]

8. Kim, K.S.; Park, W.; Hu, J.; Bae, Y.H.; Na, K. A cancer-recognizable MRI contrast agents using pH-responsive polymeric micelle. Biomaterials 2014, 35, 337-343. [CrossRef] [PubMed]

9. Xu, Q.; Zhu, L.; Yu, M.; Feng, F.; An, L.; Xing, C.; Wang, S. Gadolinium (III) chelated conjugated polymer as a potential MRI contrast agent. Polymer 2010, 51, 1336-1340. [CrossRef]

10. Zhang, L.; Liu, T.; Xiao, Y.; Yu, D.; Zhang, N. Hyaluronic Acid-Chitosan Nanoparticles to Deliver Gd-DTPA for MR Cancer Imaging. Nanomaterials 2015, 5, 1379-1396. [CrossRef]

11. Yang, W.; Guo, W.; Gong, X.; Zhang, B.; Wang, S.; Chen, N.; Yang, W.; Tu, Y.; Fang, X.; Chang, J. Facile Synthesis of Gd-Cu-In-S/ZnS Bimodal Quantum Dots with Optimized Properties for Tumor Targeted Fluorescence/MR In Vivo Imaging. ACS Appl. Mater. Inter. 2015, 7, 18759-18768. [CrossRef] [PubMed]

12. Zhang, B.; Jin, H.; Li, Y.; Chen, B.; Liu, S.; Shi, D. Bioinspired synthesis of gadolinium-based hybrid nanoparticles as MRI blood pool contrast agents with high relaxivity. J. Mater. Chem. 2012, 22, 14494-14501. [CrossRef]

13. Agatston, A.S.; Janowitz, W.R.; Hildner, F.J.; Zusmer, N.R.; Viamonte, M.; Detrano, R. Quantification of coronary artery calcium using ultrafast computed tomography. J. Am. Coll. Cardiol. 1990, 15, 827-832. [CrossRef]

14. Brenner, D.J.; Hall, E.J. Computed tomography-An increasing source of radiation exposure. New Engl. J. Med. 2007, 357, 2277-2284. [CrossRef] [PubMed]

15. Zhang, A.; Tu, Y.; Qin, S.; Li, Y.; Zhou, J.; Chen, N.; Liu, Q.; Zhang, B. Gold nanoclusters as contrast agents for fluorescent and X-ray dual-modality imaging. J. Colloid Interface Sci. 2012, 372, 239-244. [CrossRef] [PubMed]

16. Jennings, L.E.; Long, N.J. Two is better than one'-probes for dual-modality molecular imaging. Chem. Commun. 2009, 3511-3524. [CrossRef] [PubMed] 
17. Lee, S.; Chen, X. Dual-modality probes for in vivo molecular imaging. Mol. Imaging 2009, 8, 87-100. [PubMed]

18. Misri, R. Multimodality imaging. Future Med. 2013, 162-176.

19. Darder, M.; Aranda, P.; Ruiz-Hitzky, E. Bionanocomposites: A new concept of ecological, bioinspired, and functional hybrid materials. Adv. Mater. 2007, 19, 1309-1319. [CrossRef]

20. Tanaka, M.; Sato, K.; Kitakami, E.; Kobayashi, S.; Hoshiba, T.; Fukushima, K. Design of biocompatible and biodegradable polymers based on intermediate water concept. Polymer J. 2015, 47, 114-121. [CrossRef]

21. Xie, J.; Zheng, Y.; Ying, J.Y. Protein-directed synthesis of highly fluorescent gold nanoclusters. J. Am. Chem. Soc. 2009, 131, 888-889. [CrossRef] [PubMed]

22. Wang, Q.; Ye, F.; Fang, T.; Niu, W.; Liu, P.; Min, X.; Li, X. Bovine serum albumin-directed synthesis of biocompatible CdSe quantum dots and bacteria labeling. J. Colloid Interface Sci. 2011, 355, 9-14. [CrossRef] [PubMed]

23. Zhang, C.; Fu, Y.Y.; Zhang, X.; Yu, C.; Zhao, Y.; Sun, S.K. BSA-directed synthesis of CuS nanoparticles as a biocompatible photothermal agent for tumor ablation in vivo. Dalton Trans. 2015, 44, 13112-13118. [CrossRef] [PubMed]

24. Gao, F.; Cai, P.; Yang, W.; Xue, J.; Gao, L.; Liu, R.; Wang, Y.; Zhao, Y.; He, X.; Zhao, L.; et al. Ultrasmall $\left[{ }^{(64)} \mathrm{Cu}\right] \mathrm{Cu}$ Nanoclusters for Targeting Orthotopic Lung Tumors Using Accurate Positron Emission Tomography Imaging. ACS Nano 2015, 9, 4976-4986. [CrossRef] [PubMed]

25. Isab, A.A.; Hormann, A.L.; Hill, D.T.; Griswold, D.E.; DiMartino, M.J.; ShawIII, C.F. Bis(triethylphosphine)gold(I) chloride: Ionization in aqueous solution, reduction in vitro of the external and internal disulfide bonds of bovine serum albumin and antiarthritic activity. Inorg. Chem. 1989, 28, 1321-1326. [CrossRef]

26. Chen, H.; Li, S.; Li, B.; Ren, X.; Li, S.; Mahounga, D.M.; Cui, S.; Gu, Y.; Achilefu, S. Folate-modified gold nanoclusters as near-infrared fluorescent probes for tumor imaging and therapy. Nanoscale 2012, 4, 6050-6064. [CrossRef] [PubMed]

27. Arndt, B.; Noei, H.; Keller, T.F.; Müller, P.; Vonk, V.; Nenning, A.; Opitz, A.K.; Fleig, J.; Rütt, U.; Stierle, A. Structure and stability of Gd-doped $\mathrm{CeO}_{2}$ thin films on yttria-stabilized zirconia. Thin Solid Films 2016, 603, 56-61. [CrossRef]

28. Sun, G.; Zhou, L.; Liu, Y.; Zhao, Z. Biocompatible Gd III-functionalized fluorescent gold nanoclusters for optical and magnetic resonance imaging. New J. Chem. 2013, 37, 1028-1035. [CrossRef]

29. Wang, L.; Zhu, X.; Tang, X.; Wu, C.; Zhou, Z.; Sun, C.; Deng, S.-L.; Ai, H.; Gao, J. A multiple gadolinium complex decorated fullerene as a highly sensitive T(1) contrast agent. Chem. Commun. 2015, 51, 4390-4393. [CrossRef] [PubMed]

30. Zhao, Z.; Wang, X.; Zhang, Z.; Zhang, H.; Liu, H.; Zhu, X.; Li, H.; Chi, X.; Yin, Z.; Gao, J. Real-Time Monitoring of Arsenic Trioxide Release and Delivery by Activatable T(1) Imaging. ACS Nano 2015, 9, 2749-2759. [CrossRef] [PubMed]

31. Caravan, P.; Ellison, J.J.; McMurry, T.J.; Lauffer, R.B. Gadolinium(III) chelates as MRI contrast agents: Structure, dynamics, and applications. Chem. Rev. 1999, 99, 2293-2352. [CrossRef] [PubMed]

(c) 2016 by the authors; licensee MDPI, Basel, Switzerland. This article is an open access article distributed under the terms and conditions of the Creative Commons Attribution (CC-BY) license (http://creativecommons.org/licenses/by/4.0/). 